\title{
Droplet group production in an AC electro-flow focusing microdevice
}

\author{
Elena Castro-Hernández • Pablo García-Sánchez • Alfonso \\ Velencoso-Gómez • Antonio Silas-Jurado • David Fernández Rivas . \\ Antonio Ramos
}

Received: date / Accepted: date

\begin{abstract}
We report the production of droplet groups with a controlled number of drops in a microfluidic electro-flow focusing device under the action of an AC electric field. This regime appears for moderate voltages (500-700 V peak-to-peak) and signal frequencies between 25 and $100 \mathrm{~Hz}$, much smaller than the droplet production rate $(\sim 500 \mathrm{~Hz})$. For this experimental conditions the production frequency of a droplet package is twice the signal frequency. Since the continuous phase flow in the microchannel is a Hagen-Poiseuille flow, the smaller droplets of a group move faster than the bigger ones leading to droplet clustering downstream.
\end{abstract}

Keywords AC electric field · flow focusing · microfluidics · drops

\section{Introduction}

The accurate control of the volume and number of bubbles and droplets has many practical applications as,

Elena Castro-Hernández · Alfonso Velencoso-Gómez · Antonio Silas-Jurado

Área de Mecánica de Fluidos, Departamento de Ingenería Aeroespacial y Mecánica de Fluidos, Universidad de Sevilla, Avenida de los Descubrimientos s/n 41092, Sevilla, Spain.

E-mail: elenacastro@us.es

Pablo García-Sánchez · Antonio Ramos

Departamento de Electrónica y Electromagnetismo, Facultad de Física, Universidad de Sevilla, Avenida de Reina Mercedes s/n, 41012 Sevilla, Spain.

E-mail: ramos@us.es

David Fernández Rivas

Mesoscale Chemical Systems and MESA ${ }^{+}$Institute of Nanotechnology, P.O. Box 217, 7500 AE Enschede, The Netherlands. for example, in the chemical engineering, advanced materials science and biological fields (Song et al., 2006; Burns and Ramshaw, 2001; Xu et al., 2005; Garstecki et al., 2006; Ma et al., 2017; Belloul et al., 2013; Churski et al., 2012; Frenz et al., 2008). Droplet generation methods can be classified as passive or active, depending on the use of external actuation (Zhu and Wang, 2017). Techniques such as cross-flow, co-flow, flow-focusing (GañánCalvo, 1998), and step emulsification are grouped as passive. The active techniques encompass the use of electric, magnetic, or centrifugal fields, just to name a few. External forces are not only used to generate droplets ( $\mathrm{Gu}$ et al., 2008; Gañán-Calvo et al., 2006), but also to manipulate them downstream as, for example, droplet coalescence, splitting and mixing (Ray et al., 2017; Budden et al., 2013; Chokkalingam et al., 2014). Droplet cluster formation has been achieved using passive methods with a potential to serve as building blocks for new materials (Shen et al., 2016).

In this work, we generate drop clusters by combining a passive technique to produce droplets and an active one to modulate the droplet size within clusters. The former is achieved with a planar microfluidic flowfocusing junction (Anna et al., 2003), whereas the latter is accomplished by the application of an AC electric field. The advantages of using an electric field are that control is almost instantaneous and the features of drop production might be changed without altering the flow parameters (flow rate, viscosity, surface tension, etc). Furthermore, the production of droplets packages is not possible without the application of an external field. Droplet size modulation in a flow-focusing geometry has been demonstrated by using an electrical signal of triangular shape at a frequency of $10 \mathrm{~Hz}$ (He et al., 2010). In order to produce different groups of droplets we employ sinusoidal signals with frequen- 


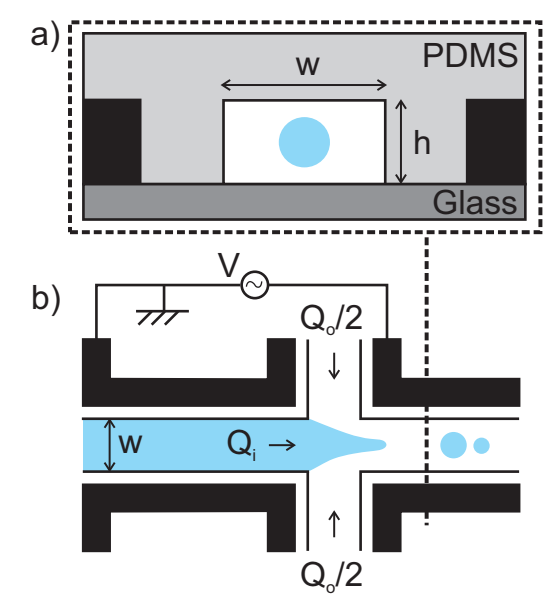

Fig. 1 a) Cross-section of the device at the level of the outlet microchannel $(w=100 \mu \mathrm{m}, h=35 \mu \mathrm{m})$. b) Sketch of a microfluidic electro-flow focusing device under an AC electric field. The electrodes are in black and the dispersed phase in blue.

cies ranging from 25 to $100 \mathrm{~Hz}$ and moderate voltages (500-700 V peak-to-peak). Clustering of droplets is carried out downstream by size-dependent velocity dispersion in the microchannel. AC electro-flow focusing devices have been previously used to control the production of droplets (Tan et al., 2014) and long jets (Castro-Hernández et al., 2015, 2016). In these works, the signal frequency was greater than the production droplet rate. Also, the existence of an unstable droplet generation regime was reported for values of the signal frequency below a threshold and sufficient voltage amplitude.

Here, we have found a new regime where generation of droplet groups with a different drop number is observed.

\section{Experimental setup}

Soft lithography techniques are used to fabricate a microfluidic electro-flow focusing device by replica molding in polydimethylsiloxane (PDMS, Dow Corning, relative permittivity $\varepsilon_{\mathrm{r}, \mathrm{PDMS}}=2.5$ ). Figure 1 shows a schematic view of the device with a rectangular crosssection $w=100 \mu \mathrm{m}$ wide and $h=35 \mu \mathrm{m}$ tall. Two sets of electrodes are patterned on both sides of the junction and they are produced using the microsolidics technique (Siegel et al., 2006). The PDMS device is plasma bonded to a glass slide. A water-in-oil (W/O) emulsion is produced by focusing an inner aqueous stream (dispersed phase) with two outer oil streams (continuous phase). The inner and outer flow rates, $Q_{\mathrm{i}}$ and $Q_{\mathrm{o}}$ respectively, are controlled by means of a double syringe pump (Model 33, Harvard Apparatus) and for all our experiments they are fixed to $Q_{\mathrm{i}}=50 \mu \mathrm{l} / \mathrm{h}$ and $Q_{\mathrm{o}}=$ $400 \mu \mathrm{l} / \mathrm{h}$. The dispersed phases are aqueous solutions of $\mathrm{KCl}$ in Milli-Q water with viscosity $\eta_{\mathrm{i}}=1 \mathrm{cP}$ and electrical conductivities $\kappa=3 \times 10^{-4} \mathrm{~S} / \mathrm{m}, \kappa=3 \times 10^{-3} \mathrm{~S} / \mathrm{m}$ and $\kappa=3 \times 10^{-2} \mathrm{~S} / \mathrm{m}$. The continuous phase is mineral oil (RTM14, Sigma Aldrich) with a viscosity of $\eta_{\mathrm{o}}=100 \mathrm{cP}$. The relative permittivity of mineral oil is $\varepsilon_{\mathrm{r}, \mathrm{o}}=2.1$ and its electrical conductivity is negligible $\left(\kappa_{\mathrm{o}}<10^{-10} \mathrm{~S} / \mathrm{m}\right)$, being considered from now on as a perfect insulator. A $5 \%(\mathrm{w} / \mathrm{w})$ of a non-ionic surfactant (Span 80, Sigma Aldrich) is added to the continuous phase lowering the surface tension of the liquid to liquid interface from $\sigma=40 \mathrm{mN} / \mathrm{m}$ to $\sigma=5 \mathrm{mN} / \mathrm{m}$, being this value independent of the $\mathrm{KCl}$ concentration (Tan et al., 2014).

The high-voltage is applied to the downstream pair of electrodes while the others are grounded, which guarantees that the incoming liquid has zero potential (CastroHernández et al., 2015, 2016). As a consequence, there is an applied AC potential difference between this incoming liquid and the downstream electrodes. A sinusoidal voltage with frequencies varying from $f=1 \mathrm{~Hz}$ to $f=1000 \mathrm{~Hz}$ (TGA1244, TTi) is amplified from $V_{\mathrm{pp}}=$ $0 \mathrm{~V}$ to $V_{\mathrm{pp}}=1000 \mathrm{~V}$ (PZD700A, Trek). The microfluidic device is placed on an inverted microscope (Eclipse Ti-U, Nikon) connected to a high-speed camera (Phantom v7.3) with a resolution of $800 \times 256 \mathrm{px}^{2}$ when operated at an acquisition rate of $10^{4} \mathrm{fps}$.

\section{Results and discussion}

Figure 2 depicts the effect of decreasing the signal frequency on the number of droplets per group for a given water conductivity $\left(\kappa=3 \times 10^{-4} \mathrm{~S} / \mathrm{m}\right)$ and voltage amplitude $\left(V_{\mathrm{pp}}=650 \mathrm{~V}\right)$. The number of droplets per group was determined by visual inspection of the videos. We performed two sets of experiments: (i) Starting from zero frequency and increasing the signal frequency; (ii) Starting from $100 \mathrm{~Hz}$ and decreasing the signal frequency. In both cases we found the same droplet groups, confirming the reproducibility and the absence of hysteresis. A greater number of droplets per group is found for smaller frequencies, and a package of $N=10$ is obtained when $f=25 \mathrm{~Hz}$. Reducing the signal frequency below this value, produces groups with a higher number of drops and an extent that exceeds the channel length. Figure 2 also shows that for $f=90 \mathrm{~Hz}$ the drop number is $N=2$ while for $f=65 \mathrm{~Hz}$ the drop number is $N=3$. For intermediate frequencies $(65-90 \mathrm{~Hz})$ groups of 2 and 3 droplets are alternatively produced. Such an intermittent production of droplet 


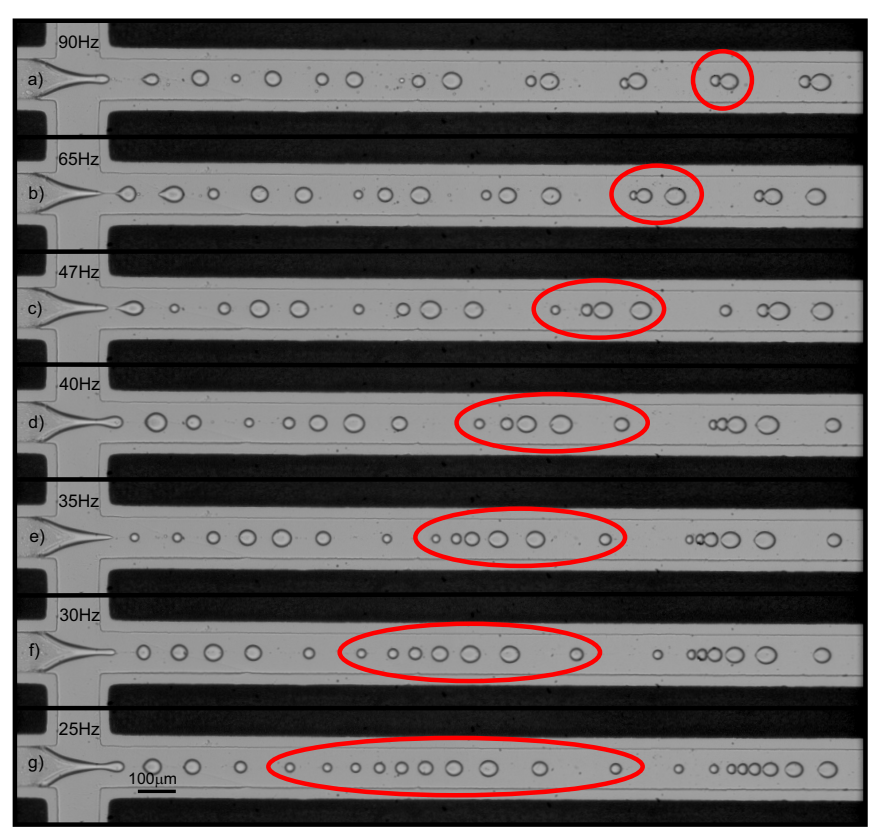

Fig. 2 Series of images showing droplet groups with a different drop number, $N$, for $Q_{\mathrm{i}}=50 \mu \mathrm{l} / \mathrm{h}, Q_{\mathrm{o}}=400 \mu \mathrm{l} / \mathrm{h}$, $\eta_{\mathrm{o}}=100 \mathrm{cP}, \kappa=3 \times 10^{-4} \mathrm{~S} / \mathrm{m}$ and $\left.V_{\mathrm{pp}}=650 \mathrm{~V}: \mathrm{a}\right) f=$ $90 \mathrm{~Hz}, N=2$; b) $f=65 \mathrm{~Hz}, N=3$; c) $f=47 \mathrm{~Hz}, N=4$; d) $f=40 \mathrm{~Hz}, N=5$; e) $f=35 \mathrm{~Hz}, N=6$; f) $f=30 \mathrm{~Hz}$, $N=7 ; \mathrm{g}) f=25 \mathrm{~Hz}, N=10$.

groups have been noticed for all in-between signal frequencies. This multi-drop regime is observed for voltage amplitudes between $V_{\mathrm{pp}}=500 \mathrm{~V}$ and $V_{\mathrm{pp}}=700 \mathrm{~V}$ and signal frequencies between $f=25 \mathrm{~Hz}$ and $f=100 \mathrm{~Hz}$. For moderate values of the voltage and signal frequencies above $f=100 \mathrm{~Hz}$ the unstable regime appears and with further increment a jet is observed (Tan et al., 2014; Castro-Hernández et al., 2015, 2016). For $V_{\mathrm{pp}}>$ $700 \mathrm{~V}$ the unstable regime is present for all tested frequencies, while for $V_{\mathrm{pp}}<500 \mathrm{~V}$ drop diameters in a group are more uniform. For fixed inner and outer flow rates, the droplet production rate is nearly constant $f_{\text {drop }} \sim 500 \mathrm{~Hz}$, only slightly dependent on signal frequency. The same trends are found for all tested water conductivities $(0.3,3$ and $30 \mathrm{mS} / \mathrm{m})$. Nevertheless, the number of droplets per group is less clear for the two highest water conductivities, presumably, because the unstable regime is more pronounced for those values. In order to check the similarity between droplet packages, we measured the drop sizes and compared them. We analyzed 1000 images for each experimental condition by using an image processing code in MATLAB. From this analysis we obtained the droplet size and calculate the polydispersity index (PDI) as in the following example: In the experimental conditions shown in Figure 2a), i.e. packages with two droplets, we obtain a PDI around 5\% for the larger drops; and for the smaller

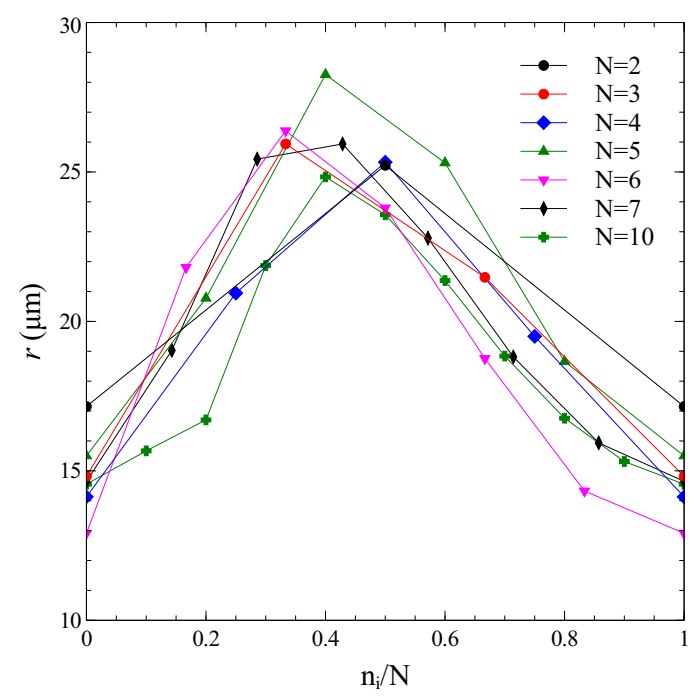

Fig. 3 Droplet radius versus relative droplet number in a package, $n_{i} / N$, for $Q_{\mathrm{i}}=50 \mu \mathrm{l} / \mathrm{h}, Q_{\mathrm{o}}=400 \mu \mathrm{l} / \mathrm{h}, \eta_{\mathrm{o}}=100 \mathrm{cP}$, $\kappa=3 \times 10^{-4} \mathrm{~S} / \mathrm{m}$ and $V_{\mathrm{pp}}=650 \mathrm{~V}$.

drops PDI is around 11\%. For all experimental conditions we obtained a PDI below $14 \%$.

Figure 3 depicts the droplet radius as a function of the relative droplet number in a package, $n_{i} / N$, where $n_{i}$ labels the droplet in the sequence of a group and $N$ is the number of droplets per group. In this figure, notice that $n_{i} / N=0$ is equivalent to $n_{i} / N=1$ due to the periodicity of droplet production. As discussed below, large droplets are produced when the signal amplitude reaches a value around zero and their size is mainly determined by the microchannel dimensions. On the other hand, the size of smaller droplets is given by the competition between electrical and capillary pressures. For the signal frequencies of this work, the meniscus should be an equipotential (at zero potential as the upstream electrodes). The charge relaxation time of our water solutions is of the order of $\varepsilon / \kappa \sim 10^{-6} \mathrm{~s}$. This time is much shorter than the signal period (of the order of $0.01 \mathrm{~s}$ ) and than the droplet breakup time (estimated from experiments as $1 / f_{d r o p}=1 / 500 \mathrm{~s}$ ). Therefore, there is enough time for charge to relax and to make the meniscus surface equipotential. The electrical stress on the surface is normal (no tangential stress) and given by $q_{\mathrm{s}}^{2} / 2 \varepsilon$, where $q_{\mathrm{s}}$ is the induced surface charge density. This induced charge is proportional to the instantaneous applied voltage, i.e. $q_{\mathrm{s}}(t) \propto V_{0} \cos (\omega t)$. Therefore, the electrical normal stress on the meniscus is

$p_{\mathrm{E}}=\frac{q_{\mathrm{s}}^{2}(t)}{2 \varepsilon} \propto \frac{V_{0}^{2}}{2}(1+\cos (2 \omega t))$,

where we have neglected the effect of the issuing drops. Otherwise, other terms should be added to the electrical 


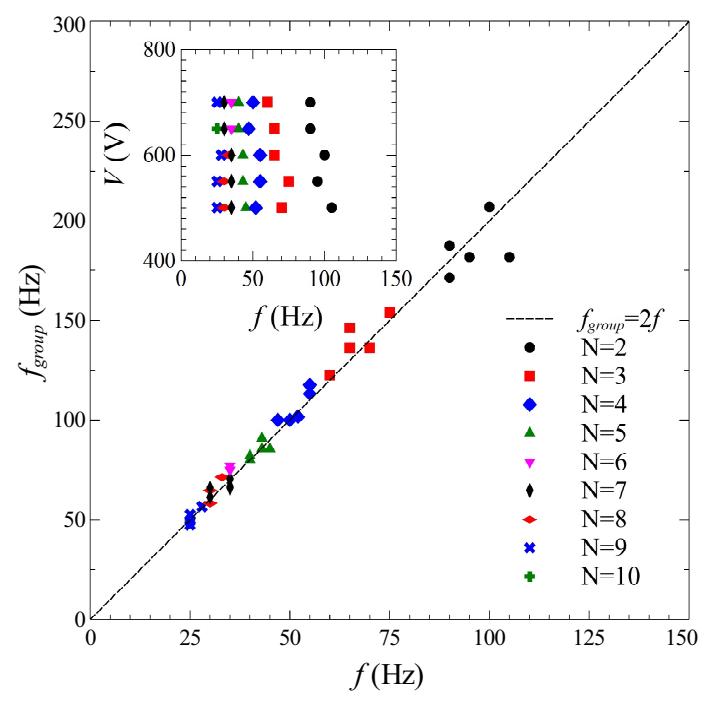

Fig. 4 Group frequency, $f_{\text {group }}$, versus signal frequency for $Q_{\mathrm{i}}=50 \mu \mathrm{l} / \mathrm{h}, Q_{\mathrm{o}}=400 \mu \mathrm{l} / \mathrm{h}, \eta_{\mathrm{o}}=100 \mathrm{cP}, \kappa=3 \times 10^{-4} \mathrm{~S} / \mathrm{m}$ and different voltages. The inset shows the number of droplets per group, $N$, as a function of voltage amplitudes and signal frequencies.

pressure. Presumably, this is the case for voltage amplitudes greater than $700 \mathrm{~V}$. Figure 3 also shows that the drop radius in a group mirrors the oscillating electrical pressure. The competition between electrical and capillary pressures suggests that smaller drops are detached for higher electrical pressure while the bigger ones are detached around zero pressure and voltage. This is in agreement with experiments using electrical signals of triangular shape (He et al., 2010).

Figure 4 displays measurements of the group frequency, $f_{\text {group }}$, versus the applied signal frequency, $f$, for $\kappa=3 \times 10^{-4} \mathrm{~S} / \mathrm{m}$ and a voltage amplitude between $V_{\mathrm{pp}}=500 \mathrm{~V}$ and $V_{\mathrm{pp}}=700 \mathrm{~V}$. The group frequency is defined as the frequency production of a droplet package. The figure also shows that the group frequency is twice the signal frequency and, thus, the droplet production rate can be determined as $f_{\text {drop }}=2 \mathrm{Nf}$. This proves that the droplet groups follow the electrical pressure given by Equation 1, consistently with the assumption of negligible electrical interaction between the drops and the meniscus. The inset in Figure 4 shows that, for our experimental conditions, the droplet number, $N$, is independent of the voltage amplitude.

We have also determined the spatial periodicity, $\lambda$, of the droplet groups for $\kappa=3 \times 10^{-4} \mathrm{~S} / \mathrm{m}$ and $V_{\mathrm{pp}}=650$ $\mathrm{V}$. This period is measured as the distance between the largest drops of two consecutive groups. Figure 5 shows a linear increase of the spatial periodicity with respect to the period of the electric forcing, $1 /(2 f)$. This seems to indicate that all groups travel at the same velocity

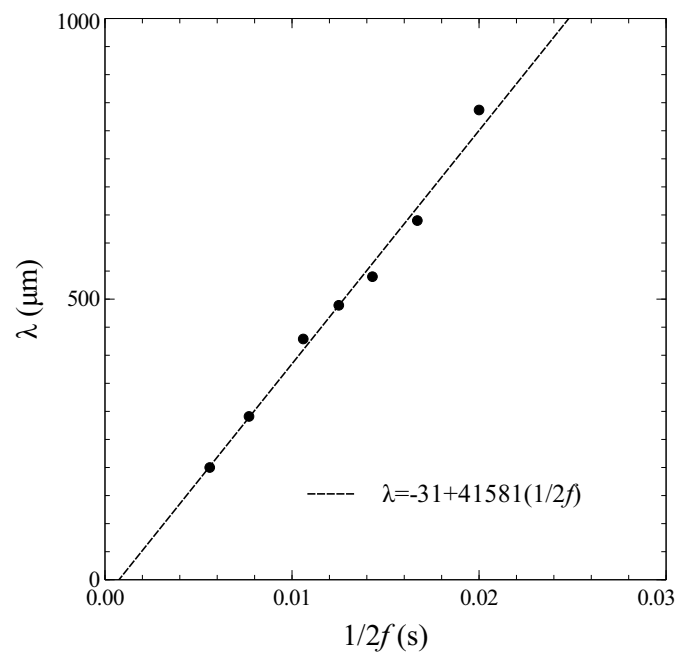

Fig. 5 Spatial periodicity versus electric forcing time for $Q_{\mathrm{i}}=50 \mu \mathrm{l} / \mathrm{h}, Q_{\mathrm{o}}=400 \mu \mathrm{l} / \mathrm{h}, \eta_{\mathrm{o}}=100 \mathrm{cP}, \kappa=3 \times 10^{-4} \mathrm{~S} / \mathrm{m}$ and $V_{\mathrm{pp}}=650 \mathrm{~V}$.

and the spatial periodicity is given by the duration of the forcing cycle. Thus, we can infer the velocity of the groups from a linear fit to the data. We have obtained $v_{\text {group }} \approx 42 \mathrm{~mm} / \mathrm{s}$, between the average and the maximum velocities within the channel, as shown below.

As the Reynolds number of the continuous phase is small, the flow in the channels is a Hagen-Poiseuille flow, with zero velocity at the walls and maximum velocity at the center of the channel. For a total flow rate of $Q_{\mathrm{o}}+Q_{\mathrm{i}}=450 \mu \mathrm{l} / \mathrm{h}$ and a rectangular channel with $h=35 \mu \mathrm{m}$ and $w=100 \mu \mathrm{m}$, the maximum flow velocity is $67.1 \mathrm{~mm} / \mathrm{s}$ and the average flow velocity is $35.7 \mathrm{~mm} / \mathrm{s}$ (Bruus, 2007). As a first approximation, the velocity of a drop is the average of the flow velocity within the volume occupied by the drop. Since drops move mainly along the axis of the channel, the smaller droplets of a group flow faster than the bigger ones and they assemble downstream of the channel. This phenomenon has been previously used to produce pairs of droplets with different sizes (Ahn et al., 2006). In our experiments, the difference in velocities between the largest and the smallest drop within a group is $\sim 5 \mathrm{~mm} / \mathrm{s}$, an order of magnitude smaller than the group velocity. Consequently, in a traveled distance of $1 \mathrm{~mm}$ the group shortens around $0.1 \mathrm{~mm}$.

\section{Conclusions}

In summary, we report the production of droplet groups with a controlled number of drops in an AC electroflow focusing microdevice. This new regime appears for 
moderate values of the voltage amplitude and signal frequencies much smaller than the droplet production rate. The production of droplet groups is only possible with the action of an external field. Also, the number of droplets per group is controlled by tuning the signal frequency, and the production rate of droplet packages is twice the signal frequency. The production of droplet groups is achieved by electrohydrodynamic means and droplet clustering is carried out by size-dependent velocity dispersion in the microchannel.

Acknowledgements The authors would like to acknowledge financial support from Spanish Government Ministry MEC under Contracts DPI2013-46485-C3-1-R and FIS2014-54539$\mathrm{P}$ and Regional Government Junta de Andalucía under Contract P11-FQM-7919. They would also like to acknowledge the technical assistance of S. Schlautman in the fabrication of the microfluidic devices.

\section{References}

Ahn K, Agresti J, Chong H, Marquez M, Weitz D (2006) Electrocoalescence of drops synchronized by size-dependent flow in microfluidic channels. Applied Physics Letters 88(26):264,105

Anna SL, Bontoux N, Stone HA (2003) Formation of dispersions using Flow Focusing in microchannels. Appl Phys Lett 82:364-366

Belloul M, Bartolo JF, Ziraoui B, Coldren F, Taly V, El Abed A (2013) High-throughput formation and control of monodisperse liquid crystals droplets driven by an alternating current electric field in a microfluidic device. Applied Physics Letters 103(3):033,112

Bruus H (2007) Theoretical microfluidics. Oxford university press Oxford

Budden M, Schneider S, Groß G, Kielpinski M, Henkel T, Köhler J (2013) Splitting and switching of microfluid segments in closed channels for chemical operations in the segment-on-demand technology. Chemical Engineering Journal 227:166 - 173

Burns JR, Ramshaw C (2001) The intensification of rapid reactions in multiphase systems using slug flow in capillaries. Lab Chip 1:10-15

Castro-Hernández E, García-Sánchez P, Tan SH, Gañán-Calvo AM, Baret JC, Ramos A (2015) Breakup length of ac electrified jets in a microfluidic flow-focusing junction. Microfluidics and Nanofluidics 19(4):787-794

Castro-Hernández E, García-Sánchez P, AlzagaGimeno J, Tan SH, Baret JC, Ramos A (2016) Ac electrified jets in a flow-focusing device: Jet length scaling. Biomicrofluidics 10(4):043,504
Chokkalingam V, Ma Y, Thiele J, Schalk W, Tel J, Huck WTS (2014) An electro-coalescence chip for effective emulsion breaking in droplet microfluidics. Lab Chip 14:2398-2402

Churski K, Kaminski TS, Jakiela S, Kamysz W, Baranska-Rybak W, Weibel DB, Garstecki P (2012) Rapid screening of antibiotic toxicity in an automated microdroplet system. Lab Chip 12:1629-1637

Frenz L, El Harrak A, Pauly M, Bégin-Colin S, Griffiths AD, Baret JC (2008) Droplet-based microreactors for the synthesis of magnetic iron oxide nanoparticles. Angewandte Chemie International Edition 47(36):6817-6820

Gañán-Calvo AM (1998) Generation of steady liquid microthreads and micron-sized monodisperse sprays in gas streams. Physical Review Letters 80(2):285

Gañán-Calvo AM, López-Herrera JM, Riesco-Chueca $\mathrm{P}$ (2006) The combination of electrospray and flow focusing. Journal of fluid Mechanics 566:421-445

Garstecki P, Fuerstman MJ, Stone HA, Whitesides GM (2006) Formation of droplets and bubbles in a microfluidic t-junction-scaling and mechanism of break-up. Lab Chip 6:437-446

Gu H, Malloggi F, Vanapalli SA, Mugele F (2008) Electrowetting-enhanced microfluidic device for drop generation. Applied Physics Letters 93(18):183,507

He P, Kim H, Luo D, Marquez M, Cheng Z (2010) Lowfrequency ac electro-flow-focusing microfluidic emulsification. Appl Phys Lett 96(17):174,103

Ma J, Lee SY, Yi C, Li CW (2017) Controllable synthesis of functional nanoparticles by microfluidic platforms for biomedical applications-a review. Lab on a Chip - Miniaturisation for Chemistry and Biology $17(2): 209-226$

Ray A, Varma V, Jayaneel P, Sudharsan N, Wang Z, Ramanujan R (2017) On demand manipulation of ferrofluid droplets by magnetic fields. Sensors and Actuators B: Chemical 242:760-768

Shen B, Ricouvier J, Malloggi F, Tabeling P (2016) Designing colloidal molecules with microfluidics. Advanced Science $3(6)$

Siegel AC, Shevkoplyas SS, Weibel DB, Bruzewicz DA, Martinez AW, Whitesides GM (2006) Cofabrication of electromagnets and microfluidic systems in poly(dimethylsiloxane). Angew Chem 118:7031-7036

Song H, Chen DL, Ismagilov RF (2006) Reactions in droplets in microfluidic channels. Angewandte chemie international edition 45(44):7336-7356

Tan SH, Semin B, Baret JC (2014) Microfluidic flowfocusing in ac electric fields. Lab Chip 14(6):10991106

Xu S, Nie Z, Seo M, Lewis P, Kumacheva E, Stone H, Garstecki P, Weibel D, Gitlin I, Whitesides G 
(2005) Generation of monodisperse particles by using microfluidics: Control over size, shape, and composition. Angewandte Chemie - International Edition 44(5):724-728

Zhu P, Wang L (2017) Passive and active droplet generation with microfluidics: a review. Lab on a Chip Miniaturisation for Chemistry and Biology 17(1):3475 